\title{
Psychische Komorbidität bei der juvenilen idiopathischen Arthritis
}

\author{
Bestandsaufnahme und Ausblick
}

\section{Mental comorbidity in juvenile idiopathic arthritis}

\author{
Review and outlook
}

Claudia Sengler ${ }^{1}$, Martina Niewerth ${ }^{1}$, Reinhard W. Holl², Reinhold Kilian ${ }^{3}$, Thomas Meissner ${ }^{4}$, Doris Staab ${ }^{5}$,

\begin{abstract}
Autoren Petra Warschburger ${ }^{6}$, Harald Baumeister ${ }^{7}$, Kirsten Minden ${ }^{1,8}$

Institute

1 Deutsches Rheuma-Forschungszentrum Berlin, Programmbereich Epidemiologie

2 Universität Ulm, Institut für Epidemiologie und medizinische Biometrie, ZIBMT

3 Universität Ulm, Klinik für Psychiatrie und Psychotherapie II

4 Universität Düsseldorf, Klinik für Kinder- und Jugendmedizin

5 Charité - Universitätsmedizin Berlin, Klinik für Klinik für Pädiatrie mit Schwerpunkt Pneumologie, Immunologie und Intensivmedizin

6 Universität Potsdam - Department Psychologie

7 Universität Ulm, Institut für Psychologie und Pädagogik

8 Charité - Universitätsmedizin Berlin, kooperatives Mitglied der Freien Universität Berlin, der Humboldt-Universität zu Berlin und des Berliner Instituts für Gesundheitsforschung, Klinik für Rheumatologie und klinische Immunologie
\end{abstract}

Schlüsselwörter

Psychische Komorbidität, Depression, Angst, juvenile idiopathische Arthritis, $\mathrm{COACH}-$ Projekt

Keywords

Mental comorbidity, depression, anxiety, juvenile idiopathic arthritis, $\mathrm{COACH}$ project

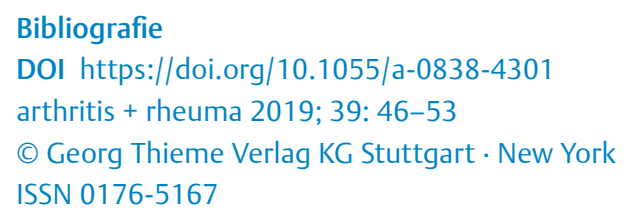

Korrespondenzadresse

Dr. Claudia Sengler

Deutsches Rheuma-Forschungszentrum Berlin

Ein Institut der Leibnizgemeinschaft

Programmbereich Epidemiologie

Charitéplatz 1, 10117 Berlin

sengler@drfz.de

\section{ZUSAMMENFASSUNG}

Psychische Erkrankungen wie Depression und Angst beginnen häufig im Jugend- und jungen Erwachsenenalter. Heranwachsende mit einer chronischen rheumatischen Erkrankung haben hierfür ein besonderes Risiko. In Untersuchungen zur psychischen Gesundheit gaben in der Regel über $10 \%$ der Jugendlichen mit juveniler idiopathischer Arthritis (JIA) depressive Symptome an. Die frühzeitige Erkennung von psychischen Belastungen ist notwendig, um Betroffenen rechtzeitig adäquate Unterstützung anbieten zu können. Geschieht das nicht, werden Möglichkeiten, die Langzeitprognose und Lebensqualität der Betroffenen zu verbessern, verpasst. Psychische Störungen werden in der klinischen Praxis oft nur am Rande berücksichtigt. Wie groß das Problem unter den bundesweit kinder- und jugendrheumatologisch betreuten Patienten mit JIA ist, wird in diesem Jahr im Rahmen des interdisziplinären Forschungsverbundes $\mathrm{COACH}$ untersucht.

\section{ABSTRACT}

Mental disorders such as depression and anxiety often begin in adolescence and young adulthood. Young people with a chronic rheumatic disease are particularly at risk. More than $10 \%$ of young people with juvenile idiopathic arthritis reported depressive symptoms in most mental health studies. Early recognition of mental health problems is necessary in order to be able to support those affected in a timely and adequate manner. If this does not happen, opportunities to improve the long-term prognosis and quality of life of the young people affected are missed. Psychological stress is often only marginally considered in clinical practice. In order to improve this, the interdisciplinary research network $\mathrm{COACH}$ will, starting this year, investigate the extent of mental health problems in adolescents and young adults with JIA, who are receiving nationwide paediatric rheumatological care. 
Mit einer Prävalenz von etwa 100 pro 100000 Kinder und Jugendliche ist die juvenile idiopathische Arthritis (JIA) die häufigste chronisch entzündliche rheumatische Erkrankung im Kindesalter [1, 2]. Schmerzen und Bewegungseinschränkungen, die mit der JIA einhergehen, können erhebliche Belastungsfaktoren darstellen, auch wenn neue Therapien, insbesondere Biologika, eine immer bessere Behandlung der JIA ermöglichen. Es gibt immer noch Patienten, die auf eine Vielzahl von Medikamenten nicht ausreichend ansprechen und daher über einen längeren Zeitraum unter Schmerzen und physischen Einschränkungen leiden. Aber auch Kinder und Jugendliche, denen mit Medikamenten und/oder ergänzenden Maßnahmen wie Physiotherapie gut geholfen werden kann, sind durch ihr Krankheitsgeschehen belastet, da sie zum Beispiel unter Nebenwirkungen der langfristig einzunehmenden Medikamente (z. B. Übelkeit bei Methotrexat-Therapie) leiden und in ihrer Freizeit durch Arztoder Physiotherapietermine eingeschränkt sein können.

Die Datenlage zu psychischen Problemen bei Kindern und Jugendlichen mit chronischen Erkrankungen ist sehr heterogen. Oftmals sind die untersuchten Patientengruppen klein und unterscheiden sich hinsichtlich des mittleren Alters, der Krankheitsdauer und -aktivität. Nicht selten werden krankheitsassoziierte Belastungsfaktoren, wie Schmerzen oder Einschränkungen in der Lebensqualität, allein durch Befragungen der Eltern eruiert, deren Angaben allerdings nicht unbedingt mit den Einschätzungen der betroffenen Kinder und Jugendlichen übereinstimmen [3]. Aussagekräftige Daten zu psychischen Belastungen können durch Befragung der Kinder und Jugendlichen selbst gewonnen werden. Es gibt eine Reihe von evaluierten Fragebögen, die eine Selbsteinschätzung durch Kinder und Jugendliche gestatten ( $\triangleright$ Tab. 1). Ergebnisse dieser Selbstbe- urteilungsfragebögen dienen nicht einer differenzierten Diagnosestellung, sondern vielmehr einem Screening auf psychische Gesundheit. Durch Identifizierung von Belastungen können Betroffene einer weiterführenden Exploration, Diagnosestellung und Therapie durch Kinder- und Jugendpsychiater oder Kinder- und Jugendlichenpsychotherapeuten zugeführt werden. Außerdem lassen standardisierte Befragungen Vergleiche zwischen verschiedenen Patienten, Patientengruppen und der allgemeinen Bevölkerung zu.

\section{Häufigkeit psychischer Belastungen bei Patienten mit JIA}

Vergleiche zwischen gesunden Menschen und Menschen mit Erkrankungen tragen zur Identifizierung krankheitsspezifischer Effekte bei. Studien, die Patienten mit JIA unter anderem auf depressive Symptome mittels Fragebögen untersuchten, sind in der Tab. 2 dargestellt.

Klinisch explorativ untersuchten Mullick et al. [4] in Bangladesch 40 Patienten mit JIA im Alter von 10 bis 18 Jahren und 40 Kontrollpersonen, die hinsichtlich Alters- und Geschlechtsverteilung vergleichbar waren. Die Autoren ermittelten eine höhere Prävalenz von psychischen Störungen, d. h. Depression, somatoformer Störung, Anpassungsstörung, gemischter Angst- und Depressionsstörung, generalisierter Angststörung, spezifischer Phobie und/oder oppositionellem Trotzverhalten, bei den Patienten mit JIA im Vergleich zur Kontrollgruppe (mindestens eine dieser Störungen bei $35 \%$ der JIA-Patienten versus 12,5\% bei den Kontrollen). Am häufigsten waren bei den JIA-Patienten Depressionen (15\%).

\ Tab. 1 Ausgewählte Instrumente zur Erfassung der patientenberichteten psychischen Gesundheit [33, 34].

\begin{tabular}{|c|c|c|c|c|c|}
\hline Instrument & $\begin{array}{l}\text { Anzahl } \\
\text { Items }\end{array}$ & Anwendbarkeit & $\begin{array}{l}\text { Schweregrad- } \\
\text { Score }\end{array}$ & $\begin{array}{l}\text { Gesamt- } \\
\text { score }\end{array}$ & Angewandte Cut offs \\
\hline $\begin{array}{l}\text { CDI: Children‘s Depression Inventory [35, 36] } \\
\text { (Kinderversion des Beck Depression Inventory) }\end{array}$ & 27 & 7-18 Jahre & $0-2$ & $0-54$ & $\begin{array}{l}\text { zwischen } 11 \text { und } 19[33] \\
\text { (Depression } \geq 17[5])\end{array}$ \\
\hline BDI: Beck Depression Inventory [37] & 21 & ab 13 Jahre & $0-3$ & $0-63$ & $\begin{array}{l}\text { zwischen } 11 \text { und } 24 \\
\text { [33] (klinisch relevante } \\
\text { Depression } \geq 18 \text { ([38]) }\end{array}$ \\
\hline BDI-FS: Beck Depression Inventory-Fast Screen [39] & 7 & ab 13 Jahre & $0-3$ & $0-21$ & $\begin{array}{l}\text { zwischen } \geq 4 \text { und } \geq 8[40] \\
\text { (Depression } \geq 4([13])\end{array}$ \\
\hline BDI: Birleson Depression Inventory [41] & 18 & ab 7 Jahre & $0-2$ & $0-36$ & Depression $\geq 13$ [11]) \\
\hline $\begin{array}{l}\text { CES-DC: Center for Epidemiological Studies Depression } \\
\text { Scale for Children [42] }\end{array}$ & 20 & $\begin{array}{l}\text { Kinder und } \\
\text { Jugendliche }\end{array}$ & $0-3$ & $0-60$ & $\begin{array}{l}\text { zwischen } 12 \text { und } 24 \text { [33] } \\
\text { (Depression } 15-16[34] \text { ) }\end{array}$ \\
\hline RADS: Reynolds Adolescent Depression Scale (43) & 30 & 13-18 Jahre & $1-4$ & $30-120$ & Zwischen 67 und 70 (33) \\
\hline MFQ: Mood and Feelings Questionnaire (44) & 33 & ab 10 Jahre & $0-2$ & $0-66$ & Depression $\geq 27[14]$ \\
\hline PHQ-9: Patient Health Questionnaire [25] & 9 & $\begin{array}{l}\text { Jugendliche und } \\
\text { Erwachsene }\end{array}$ & $0-3$ & $0-27$ & $\begin{array}{l}\text { Zwischen } \geq 5 \text { und } \geq 15[45] \\
\text { (Depression } \geq 10[25] \text { ) }\end{array}$ \\
\hline RCMAS: Revised Children's Manifest Anxiety Scale (46) & 28 & 6-19 Jahre & $0-1$ & $0-28$ & Angststörung $\geq 19$ [11]) \\
\hline $\begin{array}{l}\text { SCARED: Screen for Child Anxiety Related Emotional } \\
\text { Disorders (47) }\end{array}$ & 41 & ab 7 Jahre & $0-2$ & $0-82$ & $\begin{array}{l}\text { Angststörung } \geq 25[8] \text { oder } \\
\text { T-Score }>70[34]\end{array}$ \\
\hline GAD-7: Generalized anxiety disorder scale [26] & 7 & $\begin{array}{l}\text { Jugendliche und } \\
\text { Erwachsene }\end{array}$ & $0-3$ & $0-21$ & Angsterkrankung $\geq 10[48]$ \\
\hline
\end{tabular}


- Tab. 2 Ausgewählte Untersuchungen zur Depression bei Kindern und Jugendlichen mit JIA.

\begin{tabular}{|c|c|c|c|c|c|}
\hline & JIA-Patienten, $\mathbf{N}$ & Kontrollen, $\mathbf{N}$ & $\begin{array}{l}\text { Alter Patienten, } \\
\text { Jahre* }\end{array}$ & $\begin{array}{l}\text { Krankheitsdauer, } \\
\text { Jahre* }\end{array}$ & Depression (JIA/Kontrollen*) \\
\hline Mullick, Bangladesch, 2005 [4] & 40 & 40 & $13,25 \pm 2,75$ & $2,7 \pm 3,12$ & $\begin{array}{l}15 \% / 0 \% \\
\text { Klinischer Fragebogen (ICD-10) }\end{array}$ \\
\hline Ding, Großbritannien, 2008 [11] & 60 [65] & $* *$ & $12,3 \pm 2,8$ & $\begin{array}{l}6 \text { (6 Monate-14 Jah- } \\
\text { re) }\end{array}$ & $\begin{array}{l}\text { BDI: } 10 \pm k . A . \\
\text { BDI } \geq 14: 6,7 \% / 10 \%\end{array}$ \\
\hline Tarakci, Türkei, 2011 [8] & $52[63]$ & - & $12,13 \pm 2,92$ & $5,33 \pm 3,99$ & CDI: $8,13 \pm 4,8 / 8,44 \pm 4,85$ \\
\hline Bomba, Italien, 2013 [5] & 39 [69] & 80 & $11,43 \pm 2,1$ & $3,15 \pm 2,70$ & $\begin{array}{l}\text { CDI: } \\
8,72 \pm 6,66 / 4,99 \pm 4,05 \\
\text { CDI } \geq 17: 23 \% / 8,3 \%\end{array}$ \\
\hline Klein, Deutschland, 2018 [13] & $\begin{array}{l}50^{* * *}[72] \\
\text { JIA: } 36\end{array}$ & - & $14,1 \pm 2,3$ & k.A. & $\begin{array}{l}\text { BDI-FS } \geq 4: \\
\text { JIA } 17 \%\end{array}$ \\
\hline Hanns, Großbritannien, 2018 [14] & $102[57]$ & - & $\begin{array}{l}\text { Median } 13,2 \\
\text { (IQR } 11,9-14,2)\end{array}$ & k.A. & $\begin{array}{l}\text { Median MFQ: } 13 \text { (IQR 6- 20) } \\
\text { MFQ } \geq 27: 14,7 \%\end{array}$ \\
\hline
\end{tabular}

*Angaben in Mean \pm Standardabweichung, wenn nicht anders angegeben; ${ }^{* *}$ Normative schwedische Stichprobe für BDI [12]; ** * Patienten mit juvenilen rheumatischen Erkrankungen

Bomba et al. [5] verglich in Italien 39 Kinder mit JIA im Alter von 5 bis 16 Jahren mit 80 alters- und geschlechtsgematchten gesunden Kontrollpersonen bezüglich des Vorhandenseins bzw. der Ausprägungen von Depression und Angst sowie der gesundheitsbezogenen Lebensqualität. Sie konnten zeigen, dass die JIA-Patienten einen signifikant höheren Score im Children's Depression Inventory (CDI) aufwiesen (Mean 8,72 $\pm 6,66$ ) als die Kontrollen $(4,99 \pm 4,05 ; p=0,001)$. Weiterhin hatten 7 (23\%) der Patienten mit JIA einen CDI-Score > 17 und wiesen damit einen Risiko-Score für eine Depression auf, wohingegen dies bei den Kontrollpersonen nur $5(8,3 \%)$ waren $(P=0,041)$. Auch die Lebensqualität der JIA-Patienten, bewertet anhand des generischen Pediatric Quality of Life Inventory (PedsQL), war im Vergleich mit den Kontrollen schlechter und korrelierte mit dem Risiko für eine Depression. Außerdem unterschied sich die Beurteilung der gesundheitsbezogenen Lebensqualität (HRQoL) durch die JIA-Patienten selbst von der Einschätzung der Eltern, die eine zu den gesunden Kontrollen vergleichbare Lebensqualität in allen Skalen angaben. Diese Unterschiede in der Einschätzung von Kindern bzw. Jugendlichen und deren Eltern sind in der Literatur mehrfach beschrieben [6, 7] und unterstreichen die Bedeutung der Datenerhebung durch die betroffenen Kinder und Jugendlichen selbst.

In einer weiteren Arbeit von Tarakci et al. [8], die den CDI bei Patienten mit JIA ( $n=52$, mittleres Alter 12 Jahre, mittlere Krankheitsdauer 5 Jahre) und Kontrollpersonen anwandten, wurden die Zusammenhänge von physischer Aktivität, Angst (erhoben mit dem Screen for Child Anxiety Related Emotional Disorder [SCARED]), Depression und Funktionsfähigkeit untersucht. Dabei zeigten sich im Gegensatz zur Untersuchung von Bomba [5] keine Unterschiede im mittleren CDI von JIA-Patienten und Kontrollen sowie im SCARED. Nur in der Alltagsfunktion, bewertet anhand des Childhood Health Assessement Questionnaires (CHAQ, 0,28 \pm 0 ,24 versus $0,05 \pm 0,12$ ), stellten die Autoren signifikante Unterschiede zwischen JIA-Patienten und Kontrollen fest. Der CHAQ-Wert wiederum korrelierte signifikant mit dem CDI bei den JIA-Patienten, andere krankheitsassoziierte Parameter, wie die Anzahl der betroffenen Gelenke oder Schmerzen (Angabe auf einer visuellen Analogskala, VAS) hingegen nicht. Signifikante Assoziationen zwischen dem Ausmaß von Funktionseinbußen und der Rate an Depression wurden bereits auch in älteren Arbeiten aus den 90er-Jahren des vergangenen Jahrhunderts beschrieben $[9,10]$, in denen bei Patienten mit juveniler chronischer Arthritis mögliche Zusammenhänge zwischen Funktionsfähigkeit, weiteren krankheitsspezifischen Parametern und psychologischen bzw. psychosozialen Outcomes untersucht wurden.

In einer neueren Arbeit von Ding et al. [11] aus dem Jahr 2008 konnte ebenfalls eine Assoziation zwischen schlechterem psychologischem Outcome und funktioneller Beeinträchtigung beobachtet werden: 60 Kinder und Jugendliche mit JIA im Alter von 7 bis 18 Jahren und einer mittleren Krankheitsdauer von 6 Jahren wurden mittels Birleson Depression Inventory (BDI) sowie Revised Children`s Manifest Anxiety Scale (RCMAS) und CHAQ untersucht. Der Vergleich der Ergebnisse mit denen einer schwedischen Kontrollpopulation [12] zeigte, dass Kinder und Jugendliche mit JIA nicht häufiger unter Depressionen litten als gesunde Kontrollen; sie wiesen auch nicht häufiger Angststörungen auf. Allerdings zeigte sich eine positive Korrelation von CHAQ und BDI $(r=0,47, p<0,01)$ sowie CHAQ und RCMAS $(r=0,41, p<0,01)$, andere Parameter der Krankheitsaktivität, wie Anzahl der aktiven Gelenke, Blutsenkungsgeschwindigkeit, C-reaktives Protein oder globales Arzturteil, zeigten keine Zusammenhang mit der psychischen Gesundheit. Die Autoren argumentierten, dass sich kurzfristig ändernde Faktoren, wie Entzündungsschübe und daraus resultierende Schmerzen, als vorübergehend und damit nicht als bedrohlich wahrgenommen werden, wohingegen physische Einschränkungen, die durch den CHAQ erfasst werden, längerfristig bestehen und damit einen größeren Einfluss auf den Alltag und die psychische Gesundheit haben.

Ein etwas anderes Bild ergab sich bei einer aktuellen deutschen Untersuchung durch Klein et al. [13] an 50 Patienten im Alter von 10 bis 17 Jahren mit chronisch entzündlichen rheumatischen Er- 
krankungen, davon 36 mit einer JIA, die mit der Kurzform des Beck Depression Inventory (BDI-FS) hinsichtlich depressiver Symptome untersucht wurden. Hier zeigte sich ein Zusammenhang von Krankheitsaktivität, gemessen mittels Juvenile Arthritis Disease Activity Score-10 (JADAS-10) und dem BDI-FS-Score: JIA-Patienten mit einem Score $\geq 4$ hatten einen mittleren JADAS-10 von 10,9 (SD 7,2), während solche mit einem Score $<4$ einen mittleren JADAS- 10 von 3,6 (SD 4,1) aufwiesen ( $p=0,0002)$. Insgesamt wiesen von allen befragten Patienten 16\% Anzeichen für eine Depression, $10 \%$ gaben Suizidgedanken an.

Zusammenhänge zwischen Art, Schwere und Dauer einer chronischen Erkrankung und Depression lassen sich bei Patienten mit einer vergleichbaren Krankheitsdauer und prospektiv erhobenen Daten zum Krankheitsgeschehen analysieren; wobei Vergleiche zwischen den verschiedenen JIA-Kategorien eine ausreichende Patientenzahl voraussetzen. Hanns et al. (14) untersuchten Patienten aus der britischen JIA-Frühkohorte Childhood Arthritis Prospective Study (CAPS), in die Kinder mit JIA innerhalb von 6 Monaten nach dem ersten Kontakt zu einem pädiatrischen Rheumatologen eingeschlossen wurden, sich also noch früh im Krankheitsverlauf befanden. Daten von 102 Patienten mit den Diagnosen Polyarthritis (Rheumafaktor positiv und negativ), Oligoarthritis und Enthesitis-assoziierte Arthritis, bei denen die Baseline-Studienvisite im Alter von 11 bis 16 Jahren stattgefunden hatte und für die Ergebnisse zum Mood and Feelings Questionnaire (MFQ) innerhalb von 2 Monaten zur Einschlussuntersuchung vorlagen, konnten analysiert werden. Zu Baseline lag der mittlere MFQ-Score bei 13,0; 15 Patienten (14,7\%) wiesen einen MFQ-Score $\geq 27$, d. h. depressive Symptome, auf. Die Autoren ermittelten signifikante Zusammenhänge zwischen depressiven Symptomen mit der Zahl sowohl aktiver als auch bewegungseingeschränkter Gelenke, Schmerzen (Angabe auf visueller Analog-Skala, VAS), der Funktion (CHAQ) und der allgemeinen Beurteilung des Patienten zu seinem Krankheitsgeschehen (VAS) bei Studieneinschluss. Hinsichtlich der verschiedenen Krankheitskategorien zeigten sich folgende Unterschiede: Patienten mit Polyarthritis $(n=31)$ zeigten signifikant mehr depressive Symptome (Median MFQ-Score 19,0) als Patienten mit Oligoarthritis ( $n=53$, Median MFQ-Score 10,0; $<<0,001$ ) oder Enthesitis-assoziierter Arthritis ( $n=18$, Median MFQ-Score 9,5; $p<0,05)$. Ebenso gaben Mädchen einen signifikant höheren MFQ-Score (Median 15,5) als Jungen (Median 10,0) an ( $p<0,05)$.

Zusätzlich zu den Querschnittanalysen wurde in dieser Arbeit auch eine longitudinale Analyse mit Hilfe von gemischten linearen Modellen vorgenommen. Dabei zeigte sich, dass das Ausmaß der Depression zur Baseline-Visite stärkere Schmerzen und Funktionseinschränkungen ein Jahr später vorhersagen ließ, dann aber keine Assoziation zu der Anzahl aktiver oder bewegungseingeschränkter Gelenke mehr bestand. Dies veranschaulicht, dass es sich bei den depressiven Symptomen nicht ausschließlich um ein reaktives Geschehen auf die somatische Erkrankung handelt und daher immer gesondert erfasst und therapiert werden sollten. Interessanterweise gab es keine Assoziation von einer Therapie mit disease-modifying antirheumatic drugs (DMARDs) 12 Monate nach Einschluss in CAPS und dem Ausmaß an Depressionen zu diesem Zeitpunkt.

Die Ergebnisse dieser Studien zusammenfassend lässt sich sagen: Die Häufigkeit von depressiven Symptomen nimmt mit dem Heranwachsen zu, insbesondere bei Mädchen. Vor allem JIA-Patien- ten mit einem schweren (z. B. polyartikulären) Verlauf der Erkrankung erscheinen gefährdet. Schmerzen und Funktioneneinschränkungen waren in den meisten der hier zitieren Publikationen mit depressiven Symptomen assoziiert; in keiner Arbeit konnte jedoch ein Zusammenhang mit der Medikation festgestellt werden. Kontrovers sind bisher die Daten, ob Depression und Angst bei JIA-Patienten häufiger als in der gleichaltrigen Bevölkerung sind und wie hoch die Prävalenzrate genau ausfällt. Für derartige präzisere epidemiologische Aussagen erweist sich die Studienlage als zu heterogen und in zahlreichen Fällen als methodisch zu schwach.

\section{Häufigkeit psychische Symptome in der Bevölkerung}

Im Rahmen der Studie zur Gesundheit von Kindern und Jugendlichen in Deutschland (KiGGS) in den Jahren 2003 bis 2006 wurde auch ein Modul zur psychischen Gesundheit eingesetzt, welches Vergleichsdaten bietet: In der BELLA-Studie wurden in einer repräsentativen Subgruppe von 2863 Familien mit Kindern im Alter von 7 bis 17 Jahren Informationen mittels verschiedener Instrumente zu psychischen Problemen gesammelt $[15,16]$. Gescreent wurde auf Depression mittels Center for Epidemiological Studies Depression Scale for Children (CES-DC) und Angst mittels SCARED. Insgesamt wurde bei $10,7 \%$ der Kinder im Alter von 7 bis 10 Jahren und bei $11,1 \%$ der Jugendlichen im Alter von 11 bis 17 Jahren ein CES-DC-Score $\geq 16$ erhoben, der als Cut-off für eine manifeste Depression verwendet wurde [17]. Relevante erhöhte Score-Werte im SCARED erreichten 14,3\% der Kinder und 9,9\% der Jugendlichen. Der in der Literatur beschriebene Effekt, dass Depressionen in der Adoleszenz und dann vor allem bei Mädchen häufiger werden [18], konnte nicht gefunden werden, was möglicherweise daran liegt, dass - aus Gründen der Einheitlichkeit und damit der Vergleichbarkeit zwischen den verschiedenen Altersgruppen - nur die Eltern-Befragungen ausgewertet wurden. Tatsächlich konnte in einer weiteren Analyse aus der BELLA-Studie gezeigt werden, dass sich die Einschätzungen der Eltern und der Kinder bzw. Jugendlichen beim CES-DC unterschieden. Alle abgefragten Symptom-Komplexe (somatische Beschwerden und verzögerte Aktivität, depressiver Affekt, positiver Affekt, interpersonelle Probleme) hatten eine höhere Prävalenz in der Selbsteinschätzung durch die Kinder und Jugendlichen als in der Fremdbeurteilung durch die Eltern. Ebenso wurden Symptome eines depressiven Affekts häufiger von Mädchen als von Jungen und vor allem häufiger als von Eltern berichtet. Insgesamt wurden solche Symptome bei 11-15\% der Jungen und Mädchen von den Eltern dokumentiert; bei den Jugendlichen (11-17 Jahre) berichteten die Mädchen hingegen zu $21,7 \%$ und die Jungen zu 15,4\% von einem depressiven Affekt [19]. Weiterhin wurde ein hoher Anteil an komorbiden psychischen Problemen (z. B. von jugendlichen Mädchen selbstberichtete Angststörung in 44,6\% und problematisches Essverhalten in 47,9\%) sowie eine deutliche Einschränkung der gesundheitsbezogenen Lebensqualität bei Kindern und Jugendliche mit einem Depression-Score oberhalb des Cut-offs dokumentiert. 


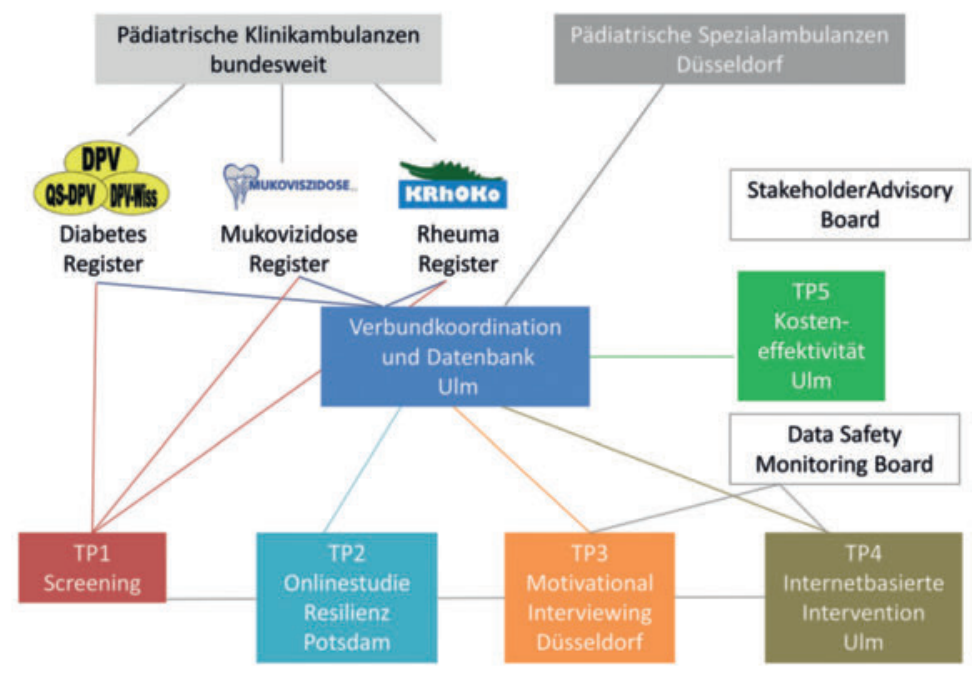

- Abb. 1 Aufbau des interdisziplinären Forschungsverbundes $\mathrm{COACH}$.

\section{Screening auf psychische Gesundheit}

Die fachspezifische Versorgung chronisch kranker Jugendlicher und junger Erwachsener ist aus verschiedenen Gründen ein wichtiger Bereich für die Früherkennung psychischer Störungen bei Jugendlichen. Kinder- und Jugendrheumatologen haben die Entwicklung eines Jugendlichen oft über Jahre hinweg begleitet und sind meist der erste Ansprechpartner bei somatischen als auch psychischen Beschwerden. Jedoch sind Kinder- und Jugendrheumatologen oft unsicher im Erkennen psychischer Beeinträchtigungen, häufig ist auch Zeitmangel ein Grund für die fehlende Exploration der Jugendlichen hinsichtlich psychischer Probleme [20]. Psychische Schwierigkeiten treten häufig in den Hintergrund der ärztlichen Untersuchung, obwohl die Prävalenz psychischer Belastungen bei jungen Menschen mit und ohne chronische Erkrankung wie gezeigt hoch relevant ist.

Ökonomisch einsetzbare und psychometrisch hochwertige Screeninginstrumente können daher eine große Hilfe bei der Erkennung psychischer Belastungen und Störungen sein. Erreicht ein Jugendlicher ein auffälliges Ergebnis in einem Screener (Summerscore über vordefinierten Cut-off-Wert), so dient dies dem Arzt als Hinweis, dass eine fachärztliche Untersuchung der Symptomatik notwendig ist. An dieser Stelle gilt es nochmals zu betonen, dass Screeningfragebögen zur Erfassung psychischer Belastungen keine differenzielle Psychodiagnostik ersetzen können, sondern vielmehr nur einen ersten Schritt in diesem Prozess darstellen.

Gerade für chronisch kranke Jugendliche und junge Erwachsene wird ein Screening auf psychische Gesundheit seit vielen Jahren gefordert (u. a. mit dem HEADSS-Interviewleitfaden für die Jugend-Sprechstunde) [21]. Zahlreiche nationale und internationale Fachgesellschaften empfehlen mittlerweile mit einem Evidenzgrad bis Ib ein systematisches jährliches Screening zur Identifizierung von Betroffenen im klinischen Alltag [22-24]. Ein entsprechend frühzeitiges Erkennen mentaler Gesundheitsprobleme ermöglicht die rechtzeitige Bereitstellung von psychotherapeutischer, psychiatrischer und verhaltensmedizinischer Interventionen und eine bessere Vermittlung Betroffener in die psychosoziale Versorgung. Dem soll nun mit dem multizentrischen Forschungsverhaben COACH (Chronic Conditions in Adolescents: Implementation and Evaluation of Patient-centred Collaborative Healthcare) Rechnung getragen werden.

\section{COACH-Projekt}

Der interdisziplinäre Forschungsverbund $\mathrm{COACH}$, dem Kinder- und Jugendmediziner, Psychologen und Psychiater aus Berlin, Düsseldorf, Potsdam und Ulm angehören ( ein gestuftes interdisziplinäres Versorgungsmodell für Jugendliche und junge Erwachsene mit ausgewählten chronischen körperlichen Erkrankungen (d. h. Typ-1-Diabetes mellitus, Mukoviszidose und JIA) und zusätzlicher psychischer Belastung anhand von fünf Teilprojekten zu entwickeln und zu evaluieren. Der Verbund wird vom Bundesministerium für Bildung und Forschung im Rahmen der „Förderung von Forschungsverbünden zur Kinder- und Jugendgesundheit“ innerhalb der „Förderinitiative Gesund - ein Leben lang“ unterstützt.

Konkret umfasst das Vorhaben ein einheitliches Screenings auf psychische Gesundheit Jugendlicher und junger Erwachsener, wofür der Patient Health Questionnaire (PHQ-9) [25] und die Generalized anxiety disorder scale (GAD-7) [26] in die nationale Diabetes-Patienten-Verlaufsdokumentation (DPV), in das Deutsche Mukoviszidose-Register und in die webbasierte Version der Kerndokumentation rheumakranker Kinder und Jugendlicher (KRhOKo) implementiert wurden. Mit dieser kurzen Befragung im Rahmen der klinischen Routine sollen Patientinnen und Patienten im Alter zwischen 12 und 21 Jahren systematisch auf depressive Störungen und Angststörungen gescreent werden (Teilprojekt 1). Im Fall positiver Screening-Befunde erhalten die Jugendlichen und ihre Familien Hinweise auf Hilfemöglichkeiten und das zusätzliche Angebot zur Teilnahme an einer Studie zur Wirksamkeit einer Internet-basierten, therapeutisch begleiteten Selbsthilfeintervention, die sich in mehreren Studien im Kinder-, Jugend- und Erwachsenenalter im 
Allgemeinen sowie bei Erwachsenen mit körperlichen Erkrankungen empirisch bereits bewährt hat (Teilprojekt 4) [27-30]. Außerdem wird im Rahmen von weiteren Teilprojekten evaluiert, welche Stärken, Fähigkeiten und Ressourcen Betroffenen geholfen haben, mit der Herausforderung einer chronischen Erkrankung umzugehen (Teilprojekt 2), inwiefern durch eine patientenzentrierte Gesprächsführung (Motivational Interviewing) die Vermittlung in die psychosoziale Unterstützung verbessert werden kann (Teilprojekt 3) [31] und welche Kosten internetbasierte Interventionsmaßnahmen hervorrufen (Teilprojekt 5).

Mithilfe einer prospektiv angelegten und online durchzuführenden Längsschnittuntersuchung sollen in Teilprojekt 2 verschiedene Forschungsfragen zur Resilienz Jugendlicher und junger Erwachsener mit einer chronischen körperlichen Erkrankung beantwortet werden. So soll beispielsweise im Rahmen dieser Studie der Frage nachgegangen werden, welche Ressourcen und Bewältigungsstrategien sich förderlich auf den Anpassungsprozess und das Wohlbefinden der betroffenen Jugendlichen auswirken. Über die Identifikation von protektiven Faktoren und Schutzmechanismen soll auch der bislang kaum untersuchten Frage, wie gute Anpassung erklärt und erreicht werden kann, nachgegangen werden.

Da Jugendliche einer Inanspruchnahme von psychologischer Unterstützung oftmals kritisch gegenüberstehen, wird im Rahmen von Teilprojekt 3 untersucht, wie betroffenen Jugendlichen der Zugang zu adäquater psychologischer Unterstützung in dieser schwierigen Situation erleichtert werden kann [32]. Der Ansatz eines Motivatonal Interviewing durch speziell geschulte Ärztinnen und Ärzte soll in einer randomisierten kontrollierten Studie mit einer Standardberatung verglichen und evaluiert werden [31]. Falls Motivational Interviewing sich für Kinder- und Jugendmediziner als wirksames Beratungsinstrument erweist und eine verbesserte Inanspruchnahme von indizierten psychologischen, psychiatrischen bzw. psychotherapeutischen Gesundheitsdienstleistungen von Jugendlichen mit chronisch körperlichen Erkrankungen und psychischen Auffälligkeiten zur Folge hat, soll diese Gesprächstechnik in der Routineversorgung etabliert werden.

Die Wirksamkeit einer internetbasierten Intervention, welche speziell für Jugendliche mit chronischen körperlichen Erkrankungen und psychischer Belastung entwickelt wurde, wird ebenfalls im Rahmen einer randomisierten kontrollierten Studie im Vergleich zu „treatment as usual“ in Teilprojekt 4 evaluiert. Die internetbasierte Intervention basiert auf etablierten Prinzipien der kognitiven Verhaltenstherapie und nutzt psychoedukative Elemente (Informationen über die psychologischen Belastungen), die individuelle Ressourcen des Jugendlichen, aktive Bewältigungsstrategien, kognitive Umstrukturierung von belastenden Gedanken, Kommunikationstraining sowie Verhaltensaktivierung zum Aufbau adaptiver Bewältigungsstrategien. Die Intervention wird mittels Informationstexten, Videos, Audioaufnahmen und therapeutischen Hausaufgaben (beispielsweise Stimmungs- und Aktivitätstagebücher) über eine gesicherte Online-Plattform durchgeführt. Die Inhalte der Intervention sind spezifisch auf die Bedürfnisse und die Lebenswelt der Jugendlichen angepasst und beziehen sich auf typische Herausforderungen und Aufgaben eines Lebens mit einer chronischen Erkrankung. Die Teilnehmenden erhalten einführend Informationen über die Funktionsweise und Handhabung der Intervention. In mehreren Modulen werden folgende Themen behandelt: Motivation und
Ressourcen, Verhaltensaktivierung, Krankheitsverständnis und -bewältigung (Angst und Depression), Emotionsregulation, Kommunikation und soziale Unterstützung, Rückfallprophylaxe sowie fakultativ auswählbare Zusatzmodule. Die Jugendlichen werden durch einen e-Coach durch das Programm geführt und erhalten individuell auf die bearbeiteten Therapieaufgaben und Hausaufgaben/ Tagebücher ein schriftliches Feedback.

Im Rahmen von Teilprojekt 5 wird erfasst, welche Versorgungsleistungen von Jugendlichen mit chronischen Erkrankungen und auffälligem Screening für Angst- und Depressionssymptome in Anspruch genommen werden. Basierend auf diesen Informationen können die entstandenen Kosten für die Volkswirtschaft und die Kostenträger abgeschätzt werden. Zusätzlich sollen in diesem Teilprojekt die Kosteneffektivität der in Teilprojekt 4 eingesetzten und auf den Prinzipien der kognitiven Verhaltenstherapie entwickelten, internetbasierten Intervention untersucht werden. Darüber können auch bedeutsame Informationen hinsichtlich der Kosteneffektivität dieser neuen Interventionsform bereitgestellt werden, die bislang für das Erwachsenenalter in begrenztem Ausmaß vorliegen, für das Kindes- und Jugendalter jedoch noch weitestgehend ausstehen.

Ziel der interdisziplinären Forschungskooperation ist es, generische Interventionskomponenten in der pädiatrischen Praxis, wie ein regelmäßiges Screening der psychischen Gesundheit, Motivationsinterviews und kognitive Verhaltenstherapie zu implementieren und zu bewerten sowie evidenzbasierte Empfehlungen für die kollaborative Gesundheitsversorgung von Jugendlichen mit chronischen Erkrankungen zu liefern.

$\mathrm{COACH}$ wird in enger Zusammenarbeit mit der Deutschen Rheuma-Liga umgesetzt. In die Entwicklung und Umsetzung aller Teilprojekte, die rheumakranke Jugendliche und junge Erwachsene betreffen, sind Forschungspartner der Deutschen Rheuma-Liga involviert.

Alle Kinder- und Jugendrheumatologen sind herzlich eingeladen, an diesem Forschungsvorhaben teilzunehmen.

\section{FAZIT}

Psychische Erkrankungen wie Depression und Angst sind häufige Begleiter einer JIA. Es gilt, sie möglichst frühzeitig zu erkennen, denn sie können langfristig die Gesundheit und Lebensqualität, das Krankheitsmanagement und damit auch die langfristigen medizinischen Behandlungsergebnisse erheblich negativ beeinflussen. Eine frühzeitige Erkennung mentaler Gesundheitsprobleme bildet die Grundlage für eine adäquate Versorgung Betroffener. Mit dem Verbundprojekt $\mathrm{COACH}$ sollen hierfür weitere Voraussetzungen geschaffen werden.

Interessenkonflikte

CS: keine; MN: keine; RK: keine; TM: keine; PW: keine; HB: keine; KM: keine 


\section{Danksagung}

Die Kerndokumentation rheumakranker Kinder und Jugendlicher wird im Jahr 2019 durch unconditional grants der Firmen Abbvie, Chugai, GSK und Novartis unterstützt; KM erhält Forschungsunterstützung durch die Deutsche Rheuma-Stiftung.

Das COACH-Vorhaben wird unter dem FKZ 01GL1440 vom BMBF gefördert.

\section{Literatur}

[1] Luque Ramos A, Hoffmann F, Albrecht K et al. Transition to adult rheumatology care is necessary to maintain DMARD therapy in young people with juvenile idiopathic arthritis. Semin Arthritis Rheum 2017; 47 (2): $269-725$

[2] Zink A, Albrecht K. Wie häufig sind muskuloskelettale Erkrankungen in Deutschland? [How frequent are musculoskeletal diseases in Germany?]. Z Rheumatol 2016; 75 (4): 346-353

[3] Garcia-Munitis P, Bandeira M, Pistorio A et al. Level of agreement between children, parents, and physicians in rating pain intensity in juvenile idiopathic arthritis. Arthritis Rheum 2006; 55 (2): 177-183

[4] Mullick MS, Nahar JS, Haq SA. Psychiatric morbidity, stressors, impact, and burden in juvenile idiopathic arthritis. J Health Popul Nutr 2005; 23 (2): 142-149

[5] Bomba M, Meini A, Molinaro A et al. Body experiences, eotional competence, and psychosocial functioning in juvenile idiopathic arthritis. Rheumatol Int 2013; 33 (8): 2045-2052

[6] Clarke SA, Eiser C. The measurement of health-related quality of life $(\mathrm{QOL})$ in paediatric clinical trials: a systematic review. Health Qual Life Outcomes.2004; 2: 66

[7] Lundberg V, Lindh V, Eriksson C et al. Health-related quality of life in girls and boys with juvenile idiopathic arthritis: self- and parental reports in a cross-sectional study. Pediatr Rheumatol Online J 2012; 10 (1): 33

[8] Tarakci E, Yeldan I, Kaya Mutlu E et al. The relationship between physical activity level, anxiety, depression, and functional ability in children and adolescents with juvenile idiopathic arthritis. Clin Rheumatol 2011; 30 (11): 1415-1420

[9] David J, Cooper C, Hickey L, et al. The functional and psychological outcomes of juvenile chronic arthritis in young adulthood. $\mathrm{Br}$ J Rheumatol 1994; 33 (9): 876-881

[10] Aasland A, Flato B, Vandvik IH. Psychosocial outcome in juvenile chronic arthritis: a nine-year follow-up. Clin Exp Rheumatol 1997; 15 (5): 561-568

[11] Ding T, Hall A, Jacobs K, David J. Psychological functioning of children and adolescents with juvenile idiopathic arthritis is related to physical disability but not to disease status. Rheumatology (Oxford) 2008; 47 (5): 660-664

[12] Ivarsson T, Gillberg C. Depressive symptoms in Swedish adolescents: normative data using the Birleson Depression Self-Rating Scale (DSRS). J Affect Disord 1997; 42 (1): 59-68

[13] Klein A, Pütz-Ata S, Kölmel T, Horneff G. Depressions-Screening in einem kinderrheumatologischen Zentrum mit dem BDI-fas-Screen. https://dgrh-kongressde/fileadmin/media/kongress/2018/Abstractbandpdf. 2018

[14] Hanns L, Cordingley L, Galloway J et al. Depressive symptoms, pain and disability for adolescent patients with juvenile idiopathic arthritis: results from the Childhood Arthritis Prospective Study. Rheumatology (Oxford) 2018; 57 (8): 1381-1389

[15] Ravens-Sieberer U, Wille N, Erhart M et al. Prevalence of mental health problems among children and adolescents in Germany: results of the
BELLA study within the National Health Interview and Examination Survey. Eur Child Adolesc Psychiatry 2008; 17 (Suppl 1): 22-33

[16] Barkmann C, Erhart M, Schulte-Markwort M, Group BS. The German version of the Centre for Epidemiological Studies Depression Scale for Children: psychometric evaluation in a population-based survey of 7 to 17 years old children and adolescents - results of the BELLA study. Eur Child Adolesc Psychiatry 2008; 17 (Suppl 1): 116-124

[17] Fendrich M, Weissman MM, Warner V. Screening for depressive disorder in children and adolescents: validating the Center for Epidemiologic Studies Depression Scale for Children. Am J Epidemiol 1990; 131 (3): 538-551

[18] Duggal S, Carlson EA, Sroufe LA, Egeland B. Depressive symptomatology in childhood and adolescence. Dev Psychopathol 2001; 13 (1): 143-164

[19] Bettge S, Wille N, Barkmann C et al., Group BS. Depressive symptoms of children and adolescents in a German representative sample: results of the BELLA study. Eur Child Adolesc Psychiatry 2008; 17 (Suppl 1): $71-81$

[20] Pietsch K, Hoyler A, Frühe B et al. Früherkennung von Depressionen in der Pädiatrie: Kriteriumsvalidität des Beck Depressions-Inventar Revison (BDI-II) und des Beck Depressions-Inventar-Fast Screen for Medical Patients (BDI-FS). Psychother Psych Med 2012; 62 (11): 418-424

[21] Cohen E, Mackenzie RG, Yates GL. HEADSS, a psychosocial risk assessment instrument: implications for designing effective intervention programs for runaway youth. J Adolesc Health 1991; 12 (7): 539-544.

[22] www.awmf.org/leitlinien/detail/11/057-016.html

[23] Cameron F], Northam EA, Ambler GR, Daneman D. Routine psychological screening in youth with type 1 diabetes and their parents: a notion whose time has come? Diabetes Care 2007; 30 (10): 2716-2724

[24] Quittner AL, Abbott J, Georgiopoulos AM et al. International Committee on Mental Health in Cystic Fibrosis: Cystic Fibrosis Foundation and European Cystic Fibrosis Society consensus statements for screening and treating depression and anxiety. Thorax 2016; 71 (1): 26-34

[25] Kroenke K, Spitzer RL, Williams JB. The PHQ-9: validity of a brief depression severity measure. J Gen Intern Med 2001; 16 (9): 606-613

[26] Lowe B, Decker O, Muller S et al. Validation and standardization of the Generalized Anxiety Disorder Screener (GAD-7) in the general population. Med Care 2008; 46 (3): 266-274

[27] Baumeister H, Grässle C, Ebert DD, Krämer LV. Blended Psychotherapy - verzahnte Psychotherapie: Das Beste aus zwei Welten? PID - Psychotherapie im Dialog 2018; 19: 51-55

[28] Domhardt M, Gesslein H, von Rezori RE, Baumeister H. Internet- and mobile-based interventions for anxiety disorders: A meta-analytic review of intervention components. Depress Anxiety 2018

[29] Bendig E, Bauereiss N, Ebert DD et al. Internet- Based Interventions in Chronic Somatic Disease. Dtsch Arztebl Int 2018; 115 (40): 659-665.

[30] Ebert DD, Van Daele T, Nordgreen T et al. Internet- and Mobile-Based Psychological Interventions: Applications, Efficacy, and Potential for Improving Mental Health

[31] A Report of the EFPA E-Health Taskforce. European Psychologist 2018; 23: $167-187$

[32] Reinauer C, Viermann R, Förtsch K et al. Motivational Interviewing as a tool to enhance access to mental health treatment in adolescents with chronic medical conditions and need for psychological support (COACH-MI): study protocol for a clusterrandomised controlled trial. Trials 2018; 19 (1): 629

[33] Gearing RE, Schwalbe CS, Dweck P, Berkowitz ]. Investigating adherence promotors in evidence-based mental health intervetnions with children and adolescents. Int J Soc Psychiatry 2013; 59 (7): 671-678

[34] Stockings E, Degenhardt L, Lee YY et al. Symptom screening scales for detecting major depressive disorder in children and adolescents: a systematic review and meta-analysis of reliability, validity and diagnostic utility. | Affect Disord 2015; 174: 447-463 
[35] Myers K, Winters NC. Ten-year review of rating scales. II: Scales for internalizing disorders. J Am Acad Child Adolesc Psychiatry 2002; 41 (6): 634-659

[36] Kovacs M. The Children's Depression Inventory (CDI). Psychopharmacol Bull 1984; 21: 995-998

[37] Helsel WJ, Matson JL. The assessment of depression in children: the internal structure of the Child Depression Inventory (CDI). Behav Res Ther 1984; 22 (3): 289-298

[38] Beck AT, Alford BA. Depression: Causes and Treatment. University of Pennsylvania PRess, University Park, PA. 2009

[39] Köllner V, Schauenburg H. Psychotherapie im Dialog - Diagnostik und Evaluation. Stuttgart - New York: Georg Thieme 2012; 38

[40] Beck AT, Brown GK, Steer RA. Beck-Depressions-Inventar-FS (BDI-FS). Manual. Deutsche Bearbeitung von Sören Kliem \& Elmar Brähler. Frankfurt am Main: Pearson Assessment, 2013

[41] Beck AT, Guth D, Steer RA, Ball R. Screening for major depression disorders in medical inpatients with the Beck Depression Inventory for Primary Care. Behav Res Ther 1997; 35 (8): 785-791

[42] Birleson P, Hudson I, Buchanan DG, Wolff S. Clinical evaluation of a self-rating scale for depressive disorder in childhood (Depression Self-Rating Scale). J Child Psychol Psychiatry 1987; 28 (1): 43-60
[43] Weissman MM, Orvaschel H, Padian N. Children's symptom and social functioning self-report scales. Comparison of mothers' and children's reports. J Nerv Ment Dis 1980; 168 (12): 736-740

[44] Reynolds W, Mazza J. Reliability and Validity of the Reynolds Adolescent Depression Scale with Young Adolescents 1998; 295-312

[45] Wood A, Kroll L, Moore A, Harrington R. Properties of the mood and feelings questionnaire in adolescent psychiatric outpatients: a research note. J Child Psychol Psychiatry 1995; 36 (2): 327-334

[46] Roseman M, Kloda LA, Saadat N et al. Accuracy of Depression Screening Tools to Detect Major Depression in Children and Adolescents: A Systematic Review. Can J Psychiatry 2016; 61 (12): 746-757

[47] Reynolds CR, Richmond BO. What I think and feel: a revised measure of children's manifest anxiety. J Abnorm Child Psychol 1978; 6 (2): 271-280

[48] Birmaher B, Khetarpal S, Brent D et al. The Screen for Child Anxiety Related Emotional Disorders (SCARED): scale construction and psychometric characteristics. J Am Acad Child Adolesc Psychiatry 1997; 36 (4): 545-553

[49] Daig I, Herschbach P, Lehmann A et al. Gender and age differences in domain-specific life satisfaction and the impact of depressive and anxiety symptoms: a general population survey from Germany. Qual Life Res 2009; 18 (6): 669-678 